\title{
Fisher information, sufficiency, and ancillarity: some clarifications
}

\author{
Nitis Mukhopadhyay · Swarnali Banerjee
}

Received: 25 July 2012 / Accepted: 11 February 2013 / Published online: 28 May 2013

(C) Sapienza Universit ̃̃ di Roma 2013

\begin{abstract}
Misconceptions are many when it comes to Fisher information, sufficiency, and ancillarity, especially among beginners. Many believe that $\mathcal{I}_{T_{1}}(\theta)+\mathcal{I}_{T_{2}}(\theta)$ should equal $\mathcal{I}_{T_{1}+T_{2}}(\theta)$ for all $\theta$. We exhibit precise scenarios where $\mathcal{I}_{T_{1}+T_{2}}(\theta)$ is $<,=$, or $>\mathcal{I}_{T_{1}}(\theta)+$ $\mathcal{I}_{T_{2}}(\theta)$ for all $\theta$. Then, we clarify the process of verifying ancillarity of a statistic with concrete examples.
\end{abstract}

Keywords Ancillarity · Dependent observations · Independent observations · Information · Sufficiency

Mathematics Subject Classification $\quad 62 \mathrm{~B} 05 \cdot 62 \mathrm{~B} 10 \cdot 62-01$

\section{Introduction}

The intriguing concepts of sufficiency and ancillarity of statistics are intertwined with the notion of information, more commonly referred to as Fisher information. We begin with a brief introduction to these notions.

Suppose that our data consist of $\mathbf{X}=\left(X_{1}, \ldots, X_{n}\right)$ having a likelihood function $L(\mathbf{x} ; \theta)$. We assume that $\mathbf{x}$ and the unknown real valued parameter $\theta$ respectively belong to some appropriate spaces $\mathcal{X}$ and $\Theta$ respectively. For simplicity, let us suppose that $\theta$ is a single parameter. Now, a statistic $T \equiv T(\mathbf{X})$, is a function of $\mathbf{X}$ alone, with $T$ 's p.m.f. or p.d.f. denoted by $g(t ; \theta)$.

N. Mukhopadhyay $(\varangle) \cdot$ S. Banerjee

Department of Statistics, University of Connecticut, Storrs, CT 06269-4120, USA

e-mail: nitis.mukhopadhyay@uconn.edu

S. Banerjee

e-mail: swarnali.banerjee@uconn.edu 
Fisher [4-6] defined the information (Fisher information) content in $\mathbf{X}$ as follows:

$$
\mathcal{I}_{\mathbf{X}}(\theta)=E_{\theta}\left[\left(\frac{\partial \ln L(\mathbf{X} ; \theta)}{\partial \theta}\right)^{2}\right]
$$

provided that customary regularity conditions hold allowing one to exchange derivative with respect to $\theta$ and integral with respect to $\mathbf{x}$. The Fisher information in a statistic $T$ is:

$$
\mathcal{I}_{T}(\theta)=E_{\theta}\left[\left(\frac{\partial \ln g(T ; \theta)}{\partial \theta}\right)^{2}\right] .
$$

Now, $T(\mathbf{X})$ is sufficient for $\theta$ if and only if the conditional distribution of $\mathbf{X}$ given $T=t$ is free from $\theta$ for all possible $t$. Equivalently, $T(\mathbf{X})$ is sufficient for $\theta$ if and only if Fisher information in $T(\mathbf{X})$ is equal to Fisher information in $\mathbf{X}$ [4-6].

Another important notion is ancillarity. A statistic $T \equiv T(\mathbf{X})$ is ancillary for $\theta$ if and only if $g(t ; \theta)$ does not involve $\theta$. That is, an ancillary statistic $T$ has no information about $\theta$. The interplay between sufficiency and ancillarity is vast, deep and complex. Recent treatments include [7,8], and other important sources cited there.

Recall that sufficiency of a statistic is often verified with the help of Neyman factorization theorem $[1,5,9,12]$ or Lehmann-Scheffé $[10]$ theorems. One may refer to a standard textbook including [13, chapter 5], [11, chapter 6], or [3, chapter 6].

While considering two statistics $T_{1}$ and $T_{2}$, there is a general scenario where one can claim that $\mathcal{I}_{T_{1}+T_{2}}(\theta)=\mathcal{I}_{T_{1}}(\theta)+\mathcal{I}_{T_{2}}(\theta)$ for all $\theta$. We claim:

$$
\mathcal{I}_{\left(T_{1}, T_{2}\right)}(\theta)=\mathcal{I}_{T_{1}}(\theta)+\mathcal{I}_{T_{2}}(\theta) \text { when } \mathrm{T}_{1}, \mathrm{~T}_{2} \text { are independent, }
$$

and additionally

$$
\mathcal{I}_{T_{1}+T_{2}}(\theta)=\mathcal{I}_{\left(T_{1}, T_{2}\right)}(\theta) \text { when } \mathrm{T}_{1}+\mathrm{T}_{2} \text { is sufficient. }
$$

Combining (3) and (4), we conclude $\mathcal{I}_{T_{1}+T_{2}}(\theta)=\mathcal{I}_{T_{1}}(\theta)+\mathcal{I}_{T_{2}}(\theta)$ for all $\theta$ when (i) $T_{1}, T_{2}$ are independently distributed and (ii) $T_{1}+T_{2}$ is a sufficient statistic.

Our aim is two-fold. Many beginners (students in introductory mathematical statistics courses) believe that $\mathcal{I}_{T_{1}+T_{2}}(\theta)=\mathcal{I}_{T_{1}}(\theta)+\mathcal{I}_{T_{2}}(\theta)$ for any two statistics $T_{1}, T_{2}$. This misconception often adversely affects the grade of many students in assignments and exams. Hence, we first give concrete examples to show that such a result may not hold generally. The Fisher information in the sum of two statistics $T_{1}, T_{2}$ may be equal, less than, or greater than the sum of the individual Fisher information for all $\theta$ so that one should proceed carefully before claiming whether or not $\mathcal{I}_{T_{1}+T_{2}}(\theta)=\mathcal{I}_{T_{1}}(\theta)+\mathcal{I}_{T_{2}}(\theta)$.

In introductory courses on mathematical statistics, one is exposed to an important result which is customarily referred to as Basu's [2] theorem. Simply put, Basu's theorem proved that a complete and sufficient statistic $S$ would be distributed independently of an ancillary statistic $T$. Since independent statistics often play major roles in many problems of statistical inference, Basu's theorem holds a key position in all of it. But, we find that many beginners get amply confused about how to show rigorously if a statistic $T$ is ancillary before they may proceed to apply Basu's theorem.

In Sect. 3, we clarify the process of determining ancillarity of a statistic. We emphasize that a statistic $T$ is ancillary for $\theta$ if it can be expressed as a function of other random variables $U_{1}, \ldots, U_{k}$ whose joint distribution does not involve $\theta$. Section 4 gives some concluding thoughts. 


\section{Fisher information in a sum of statistics may be equal to, smaller than or larger than the sum of the individual Fisher information}

How does Fisher Information $\mathcal{I}_{T_{1}+T_{2}}(\theta)$ in the sum of statistics $T_{1}, T_{2}$ compare with $\mathcal{I}_{T_{1}}(\theta)+$ $\mathcal{I}_{T_{2}}(\theta)$ ? Its answer may be all over the place. But our experience is that many beginners feel rather confident to first "simplify" $\mathcal{I}_{T_{1}+T_{2}}(\theta)$ as $\mathcal{I}_{T_{1}}(\theta)+\mathcal{I}_{T_{2}}(\theta)$, thereby evaluating $\mathcal{I}_{T_{1}+T_{2}}(\theta)$ by adding $\mathcal{I}_{T_{1}}(\theta), \mathcal{I}_{T_{2}}(\theta)$.

We want to clarify such a misconception. The following examples will highlight specific scenarios where $\mathcal{I}_{T_{1}+T_{2}}(\theta)$ may be equal to, less than, or greater than $\mathcal{I}_{T_{1}}(\theta)+\mathcal{I}_{T_{2}}(\theta)$. That is, one should proceed with great caution before one equates $\mathcal{I}_{T_{1}+T_{2}}(\theta)$ with $\mathcal{I}_{T_{1}}(\theta)+\mathcal{I}_{T_{2}}(\theta)$.

Example 2.1 Suppose that $T_{1} \sim N(\theta, 1), T_{2} \sim N(\theta, 4)$ and they are independent. Here, $\theta$ is an unknown parameter which is a real number. Clearly, $\mathcal{I}_{T_{1}}(\theta)=1$ and $\mathcal{I}_{T_{2}}(\theta)=\frac{1}{4}$. But, $T_{1}+T_{2} \sim N(2 \theta, 5)$ or equivalently $\frac{1}{2}\left(T_{1}+T_{2}\right) \sim N\left(\theta, \frac{5}{4}\right)$ so that $\mathcal{I}_{T_{1}+T_{2}}(\theta)=$ $\mathcal{I}_{\frac{1}{2}\left(T_{1}+T_{2}\right)}(\theta)=\frac{4}{5}<\mathcal{I}_{T_{1}}(\theta)+\mathcal{I}_{T_{2}}(\theta)$ for all $\theta$.

Example 2.2 In Example 2.1, one may quickly argue by factorization of the likelihood function that $T_{1}+T_{2}$ is not sufficient for $\theta$ and one may get an impression that non-sufficiency of $T_{1}+T_{2}$ led to $\mathcal{I}_{T_{1}+T_{2}}(\theta)<\mathcal{I}_{T_{1}}(\theta)+\mathcal{I}_{T_{2}}(\theta)$. We highlight a situation where $T_{1}+T_{2}$ is not sufficient, but $\mathcal{I}_{T_{1}+T_{2}}(\theta)=\mathcal{I}_{T_{1}}(\theta)+\mathcal{I}_{T_{2}}(\theta)$. Consider $T_{1}, T_{2}, T_{3}$ distributed independently as $\operatorname{Bernoulli}(\theta)$ where $0<\theta<1$ is an unknown parameter. Then, we can obviously claim:

$$
\mathcal{I}_{T_{1}+T_{2}}(\theta)=\mathcal{I}_{T_{1}}(\theta)+\mathcal{I}_{T_{2}}(\theta),
$$

for all $\theta$, but $T_{1}+T_{2}$ is not sufficient for $\theta$ since $T_{3}$ has been left out.

Remark 2.1 Example 2.2 may have introduced a tricky situation by indirectly raising a larger issue of whether we ever have sufficiency! In principle, we could go out and gather more data rendering our current data non-sufficient. That is true, however, the reassurance comes from the most basic and fundamental understanding that the classical notion of sufficiency and closely related principles of inference assume the customary sample size in the context of our current data known, arbitrary, but held fixed.

Example 2.3 Suppose that $\left(T_{1}, T_{2}\right) \sim N_{2}(0,0,1,1, \theta)$, a bivariate normal distribution, with the correlation coefficient $\theta$ unknown, $\theta \in(-1,1)-\{0\}$. We note that $T_{1}+T_{2}$ is not sufficient for $\theta$. Clearly, $T_{1} \sim N(0,1), T_{2} \sim N(0,1)$, and $T_{1}+T_{2} \sim N(0,2+2 \theta)$. Thus, $\mathcal{I}_{T_{1}}(\theta)=\mathcal{I}_{T_{2}}(\theta)=0$, but $\mathcal{I}_{T_{1}+T_{2}}(\theta)$ is surely positive. Then, $\mathcal{I}_{T_{1}+T_{2}}(\theta)>\mathcal{I}_{T_{1}}(\theta)+\mathcal{I}_{T_{2}}(\theta)$ for all $\theta$.

Example 2.4 Example 2.3 may not greatly appeal to some readers because it had $\mathcal{I}_{T_{1}}(\theta)=$ $\mathcal{I}_{T_{2}}(\theta)=0$ for all $\theta$. So, we give an example where $\mathcal{I}_{T_{1}}(\theta)>0, \mathcal{I}_{T_{2}}(\theta)>0$ for all $\theta$, but $\mathcal{I}_{T_{1}+T_{2}}(\theta)>\mathcal{I}_{T_{1}}(\theta)+\mathcal{I}_{T_{2}}(\theta)$ for all $\theta$. Suppose that $\left(T_{1}, T_{2}\right) \sim N_{2}(\theta, 2 \theta, 1,4, \rho)$ where $\theta$ is an unknown real number and $\rho$ is known, $\rho \in\left(-1,-\frac{1}{8}\right)$. We note that $T_{1}+T_{2}$ is not sufficient for $\theta$. Clearly, $T_{1} \sim N(\theta, 1), T_{2} \sim N(2 \theta, 4)$, and $T_{1}+T_{2} \sim N(3 \theta, 5+4 \rho)$ so that $\mathcal{I}_{T_{1}}(\theta)=1, \mathcal{I}_{T_{2}}(\theta)=\mathcal{I}_{\frac{1}{2} T_{2}}(\theta)=1$, but $\mathcal{I}_{T_{1}+T_{2}}(\theta)=\mathcal{I}_{\frac{1}{3}\left(T_{1}+T_{2}\right)}(\theta)=\frac{9}{5+4 \rho}$ for all $\theta$. Then, $\mathcal{I}_{T_{1}+T_{2}}(\theta)>\mathcal{I}_{T_{1}}(\theta)+\mathcal{I}_{T_{2}}(\theta)$ for all $\theta$.

Example 2.5 (Example 2.4 continued). Let $\left(T_{1}, T_{2}\right) \sim N_{2}(\theta, 2 \theta, 1,4, \rho)$ where $\theta$ is an unknown real number and $\rho$ is known, $\rho \in\left(-\frac{1}{8}, 1\right)-\{0\}$. Then, $\mathcal{I}_{T_{1}+T_{2}}(\theta)<\mathcal{I}_{T_{1}}(\theta)+\mathcal{I}_{T_{2}}(\theta)$ for all $\theta$. 
Table 1 A Summary from Examples 2.1 - 2.6: $A=\mathcal{I}_{T_{1}+T_{2}}(\theta)$ and $B=\mathcal{I}_{T_{1}}(\theta)+\mathcal{I}_{T_{2}}(\theta)$

\begin{tabular}{llllll}
\hline Example\# & $\begin{array}{l}\text { Are } \\
T_{1}, T_{2} \\
\text { independent? }\end{array}$ & $\begin{array}{l}\text { Are } T_{1}, T_{2} \\
\text { identically } \\
\text { distributed? }\end{array}$ & $\begin{array}{l}\text { Is } \\
T_{1} \text { or } T_{2} \\
\text { ancillary? }\end{array}$ & $\begin{array}{l}\text { Is } \\
T_{1}+T_{2} \\
\text { sufficient? }\end{array}$ & $\begin{array}{l}\text { Is } A<, \\
=, \text { or }>B ?\end{array}$ \\
\hline 2.1 & Yes & No & No & No & $<$ \\
2.2 & Yes & Yes & No & No & $=$ \\
2.3 & No & Yes & Yes, both & No & $>$ \\
2.4 & No & No & No & No & $>$ \\
2.5 & No & No & No & No & $<$ \\
2.6 & No & No & No & Yes & $>$ \\
\hline
\end{tabular}

Example 2.6 In each previous example, $T_{1}+T_{2}$ was non-sufficient for $\theta$. In the present example, we have $T_{1}+T_{2}$ sufficient for $\theta$, but $\mathcal{I}_{T_{1}+T_{2}}(\theta)$ and $\mathcal{I}_{T_{1}}(\theta)+\mathcal{I}_{T_{2}}(\theta)$ are not equal for any $\theta$. We begin with $Y_{1}, Y_{2}$ independent, both distributed as $N(\theta, 1)$. Here, $\theta$ is an unknown parameter which is a real number. We define $T_{1}=Y_{1}-2 Y_{2}$ and $T_{2}=Y_{1}+4 Y_{2}$. Since $T_{1} \sim N(-\theta, 5), T_{2} \sim N(5 \theta, 17)$, we have $\mathcal{I}_{T_{1}}(\theta)=\frac{1}{5}, \mathcal{I}_{T_{2}}(\theta)=\frac{25}{17}$. But, $T_{1}+T_{2} \sim$ $N(4 \theta, 8)$ so that $\mathcal{I}_{T_{1}+T_{2}}(\theta)=2$. Obviously, $T_{1}+T_{2}\left(=2\left(Y_{1}+Y_{2}\right)\right)$ is sufficient for $\theta$. But, $\mathcal{I}_{T_{1}}(\theta)+\mathcal{I}_{T_{2}}(\theta)=1 \frac{57}{85}<\mathcal{I}_{T_{1}+T_{2}}(\theta)$ for all $\theta$.

Table 1 succinctly summarizes the different stories told by our examples. One will observe that under comparable scenarios, Examples 2.4 and 2.5 give different conclusions.

\section{How to prove if a statistic $T$ is ancillary?}

Ancillary statistics play crucial roles in statistical inference. For an overview, see [7,8], and other important sources cited there.

It is important to check if a statistic is ancillary and that is our sole motivation in this section. How does one verify ancillarity? Often a statistic $T$ may be expressed as a function of other random variables $U_{1}, \ldots, U_{k}$ where the marginal distribution of each $U_{i}$ is free from $\theta$. But, this in itself may not guarantee that $T$ is ancillary.

We emphasize that a statistic $T$ is ancillary if and only if $T$ is a function of other random variables $U_{1}, \ldots, U_{k}$ whose joint distribution does not involve $\theta$. We show the importance of this result with the help of some examples. Indeed, the distribution of a generic random variable $W$ is free from $\theta$ if and only if $W$ is a function of some other random variables $U_{1}, \ldots, U_{k}$ whose joint distribution does not involve $\theta$. The following examples will illustrate and strengthen this basic idea.

Example 3.1 Let $X_{1}, X_{2}$ be jointly distributed as $N_{2}\left(\theta, \theta, 1,1,\left(\theta^{2}+1\right)^{-1}\right)$ where $\theta$ is an unknown parameter, $-\infty<\theta<\infty$. The means and variances will have appropriate units whereas the correlation will have no unit. Just because we denote the means $\theta$, for example expressed in seconds, we obviously do not imply that the correlation has a unit like $\mathrm{sec}^{-2}$. It is just that this correlation takes into account the $\theta^{2}$ value in its expression and it is obviously unit-free.

The marginal distribution of each $X_{1}, X_{2}$ is $N(\theta, 1)$. Now, consider a statistic $T=X_{1}-$ $X_{2} \equiv U_{1}-U_{2}$ where $U_{i}=X_{i}-\theta$. Here, the fact that marginally $U_{1}, U_{2}$ are both standard normal random variables is not enough to conclude ancillarity (if it is so) of $T$. Note that 
the joint distribution of $U_{1}, U_{2}$ is $N_{2}\left(0,0,1,1,\left(\theta^{2}+1\right)^{-1}\right)$ which involves $\theta$. Indeed, $T \sim$ $N\left(0,2 \theta^{2}\left(\theta^{2}+1\right)^{-1}\right)$ so that $T$ cannot be an ancillary statistic.

Example 3.2 In Example 3.1, the random variables $X_{1}$ or $X_{2}$ were not individually ancillary for $\theta$, but the distribution of $X_{1}-X_{2}$ involved $\theta$. Now, suppose that $X_{1}, X_{2}$ are jointly distributed as $N_{2}(0,0,1,1, \theta)$ where $\theta$ is an unknown parameter, $-1<\theta<1$. Here, $X_{1}$ is an ancillary statistic for $\theta$, and so is $X_{2}$. This sets this example apart from Example 3.1. Yet, $T=X_{1}-X_{2}$ is not an ancillary statistic for $\theta$ since $T \sim N(0,2(1-\theta))$ that involves $\theta$.

Example 3.3 This example is more involved. Suppose that $X_{1}, X_{2}, X_{3}$ are jointly distributed as a trivariate normal with the respective mean vector and dispersion matrix as follows:

$$
\left(\begin{array}{l}
\theta \\
\theta \\
\theta
\end{array}\right) \quad \text { and } \quad \theta^{2}\left(\begin{array}{lll}
1 & \theta & \theta^{2} \\
\theta & 1 & \theta \\
\theta^{2} & \theta & 1
\end{array}\right)
$$

where $\theta$ is an unknown parameter, $-1<\theta<1$. In the sense that we have explained in Example 3.1, there is no abuse or mix-up of units while writing down the covariance matrix in (5). More precisely, we write the means $\theta$, for example in seconds, whereas $\operatorname{Cov}\left(X_{1}, X_{2}\right)=$ $\theta^{3} \sec ^{2}, \operatorname{Cov}\left(X_{1}, X_{3}\right)=\theta^{4} \sec ^{2}, \operatorname{Cov}\left(X_{2}, X_{3}\right)=\theta^{3} \sec ^{2}$.

The question is whether the statistic $T=\left(X_{1}-X_{2}\right) /\left(X_{2}-X_{3}\right)$ is ancillary. Let us define $W_{i}=X_{i}-\theta$ so that $X_{1}-X_{2} \equiv W_{1}-W_{2}$ and $X_{2}-X_{3} \equiv W_{2}-W_{3}$ where both $W_{1}-W_{2}, W_{2}-W_{3}$ are marginally distributed as $N_{2}\left(0,2 \theta^{2}(1-\theta)\right)$. But, we can express $T=U_{1} / U_{2}$ where $U_{1}=\left(W_{1}-W_{2}\right) / \sqrt{2 \theta^{2}(1-\theta)}$ and $U_{2}=\left(W_{2}-W_{3}\right) / \sqrt{2 \theta^{2}(1-\theta)}$, both standard normal random variables. However, this does not imply that $T$ is ancillary.

Observe that $U_{1}, U_{2}$ are jointly distributed as $N_{2}(0,0,1,1, \rho)$ with the correlation coefficient $\rho \equiv \rho(\theta)=-\frac{1}{2}(1-\theta)$. Now, the distribution of $T=U_{1} / U_{2}$ corresponds to the Cauchy pd.f.:

$$
g(t ; \theta)=\frac{\sqrt{1-\rho^{2}}}{\pi\left\{\left(1-\rho^{2}\right)+(t-\rho)^{2}\right\}},-\infty<t<\infty,
$$

which involves $\theta$ so that $T$ is not ancillary.

\section{Some final thoughts}

Intricate concepts of sufficiency, ancillarity, and Fisher information are not easy to master. The internal threads connecting these concepts often overwhelm the beginners leading to misconceptions. With the help of simple but concrete examples we have emphasized certain specific issues surrounding Fisher information in the sum of statistics and the determination of ancillarity of a statistic.

It is our belief that many beginners will appreciate this discourse. At the same time, some teachers may readily integrate these illustrations in classroom instructions to strengthen students' basic understanding of fundamental concepts such as sufficiency, ancillarity, and Fisher information.

In this discourse, we restrict attention to a single real-valued parameter of interest for the sake of brevity. Generally speaking, however, our $\theta$ may be a vector parameter that includes all parameters in a likelihood function, nuisance or not. 
Acknowledgments We appreciated helpful feedback from the editor and a referee. We thank them both.

\section{References}

1. Bahadur, R.R.: Sufficiency and statistical decision functions. Ann. Math. Stat. 25, 423-462 (1954)

2. Basu, D.: On statistics independent of a complete sufficient statistic. Sankhyā 15, 377-380 (1955)

3. Casella, G., Berger, R.L.: Statistical inference, 2nd edn. Duxbury, California (2002)

4. Fisher, R.A.: A mathematical examination of the methods of determining the accuracy of an observation by the mean error, and by the mean square error. Mon. Not. R. Astron. Soc. 80, 758-770 (1920)

5. Fisher, R.A.: On the mathematical foundations of theoretical statistics. Philos. Trans. R. Soc. A222, 309-368 (1922)

6. Fisher, R.A.: Theory of statistical estimation. Proc. Camb. Philos. Soc. 22, 700-725 (1925)

7. Ghosh, M.: Basu's theorem with applications: a personalistic review. Sankhyā Ser A 64, 509-531 (2002)

8. Ghosh, M., Fraser, D.A.S., Reid, N.: Ancillarity statistics: a review. Stat. Sin. 20, 1309-1322 (2010)

9. Halmos, P.R., Savage, L.J.: Application of the Radon-Nikodym theorem to the theory of sufficient statistics. Ann. Math. Stat. 20, 225-241 (1949)

10. Lehmann, E.L., Scheffé, H.: Completeness, similar regions and unbiased estimation-part I. Sankhyā 10, 305-340 (1950)

11. Mukhopadhyay, N.: Probability and statistical inference. Dekker, New York (2000)

12. Neyman, J.: Sur un teorema concernente le cosiddette statistiche sufficienti. Giornale dell Instituto Italiano degli Attuari 6, 320-334 (1935)

13. Rao, C.R.: Linear statistical inference and its applications, 2nd edn. Wiley, New York (1973) 